\title{
Macroscopic Dynamics of the Strong-Coupling BCS-Hubbard Model
}

\author{
J.-B. Bru W. de Siqueira Pedra
}

May 27, 2021

\begin{abstract}
The aim of the current paper is to illustrate, in a simple example, our recent, very general, rigorous results $[1,2]$ on the dynamical properties of fermions and quantum-spin systems with long-range, or mean-field, interactions, in infinite volume. We consider here the strongcoupling BCS-Hubbard model studied in $[3,4]$, because this example is very pedagogical and, at the same time, physically relevant for it highlights the impact of the (screened) Coulomb repulsion on ( $s$-wave) superconductivity.
\end{abstract}

Keywords: superconductivity, BCS, Hubbard, quantum dynamics.

\section{Presentation of the Model}

The most general form of a translation invariant model for fermions with two-body interactions in a cubic box $\Lambda_{L} \doteq\{\mathbb{Z} \cap[-L, L]\}^{d}$ (d-dimensional crystal) of volume $\left|\Lambda_{L}\right|, L \in \mathbb{N}_{0}$, is given in momentum space by

$$
\mathrm{H}_{L}^{F u l l}=\sum_{k \in \Lambda_{L}^{*}, \mathrm{~s} \in \mathrm{S}}\left(\varepsilon_{k}-\mu\right) \tilde{a}_{k}^{*} \tilde{a}_{k}+\frac{1}{\left|\Lambda_{L}\right|} \sum_{\substack{k, k^{\prime}, q \in \Lambda_{L}^{*} \\ \mathrm{~s}_{1}, \mathrm{~s}_{2}, \mathrm{~s}_{3}, \mathrm{~s}_{4} \in \mathrm{S}}} g_{\mathrm{s}_{1}, \mathrm{~s}_{2}, \mathrm{~s}_{3}, \mathrm{~s}_{4}}\left(k, k^{\prime}, q\right) \tilde{a}_{k+q, \mathrm{~s}_{1}}^{*} \tilde{a}_{k^{\prime}-q, \mathrm{~s}_{2}}^{*} \tilde{a}_{k^{\prime}, \mathrm{s}_{3}} \tilde{a}_{k, \mathrm{~s}_{4}} .
$$

See [5, Eq. (2.1)]. Here, $\mathrm{S}$ is some finite (spin) set representing the internal degrees of freedom of quantum particles and $\Lambda_{L}^{*}$ is the reciprocal lattice of quasi-momenta (periodic boundary conditions) associated with $\Lambda_{L}$. The operator $\tilde{a}_{k, \mathrm{~s}}^{*}$ (respectively $\tilde{a}_{k, \mathrm{~s}}$ ) creates (respectively annihilates) a fermion with spin s $\in \mathrm{S}$ and (quasi-) momentum $k \in \Lambda_{L}^{*}$, the function $\varepsilon_{k}$ represents the kinetic energy of a fermion with (quasi-) momentum $k$ and the real number $\mu$ is the chemical potential. The last term of (1) corresponds to a translation-invariant two-body interaction written in the momentum space.

One important example of a fermionic system with long-range interactions is given in the scope of the celebrated BCS theory - proposed in the late 1950s (1957) to explain conventional type I superconductors. The lattice version of this theory is obtained from (1) by taking $S \doteq\{\uparrow, \downarrow\}$ and imposing

$$
g_{\mathrm{s}_{1}, \mathrm{~s}_{2}, \mathrm{~s}_{3}, \mathrm{~s}_{4}}\left(k, k^{\prime}, q\right)=\delta_{k,-k^{\prime}} \delta_{\mathrm{s}_{1}, \uparrow} \delta_{\mathrm{s}_{2}, \downarrow} \delta_{\mathrm{s}_{3}, \downarrow} \delta_{\mathrm{s}_{4}, \uparrow} f(k,-k, q)
$$

for some function $f$ : It corresponds to the so-called (reduced) BCS Hamiltonian

$$
\mathrm{H}_{L}^{B C S} \doteq \sum_{k \in \Lambda_{L}^{*}}\left(\varepsilon_{k}-\mu\right)\left(\tilde{a}_{k, \uparrow}^{*} \tilde{a}_{k, \uparrow}+\tilde{a}_{k, \downarrow}^{*} \tilde{a}_{k, \downarrow}\right)-\frac{1}{\left|\Lambda_{L}\right|} \sum_{k, q \in \Lambda_{L}^{*}} \gamma_{k, q} \tilde{a}_{k, \uparrow}^{*} \tilde{a}_{-k, \downarrow}^{*} \tilde{a}_{-q, \downarrow} \tilde{a}_{q, \uparrow}
$$


where $\gamma_{k, q}$ is a positive ${ }^{1}$ function. Because of the term $\delta_{k,-k^{\prime}}$, the interaction of this model has a long-range character, in position space. The simple choice $\gamma_{k, q}=\gamma>0$ in (2) is still physically very interesting since, even when $\varepsilon_{k}=0$, the BCS Hamiltonian qualitatively displays most of basic properties of real conventional type I superconductors. See, e.g. [6, Chapter VII, Section 4]. The case $\varepsilon_{k}=0$ is known as the strong coupling limit of the BCS model. The dynamical properties of the BCS Hamiltonian $\mathrm{H}_{L}^{B C S}$ with $\gamma_{k, q}=\gamma>0$ can be explicitly computed from results of [1,2], but we prefer here to consider another BCS-type model including the Hubbard interaction, this being a much richer example.

An important physical fact not taken into account in the BCS theory is the Coulomb interaction between electrons or holes, which can imply strong correlations, like in cuprates with the universally observed Mott transition at zero doping. This problem was of course already addressed in theoretical physics right after the emergence of the Fröhlich model and the BCS theory, see, e.g., [7].

We present below a model, named here the strong-coupling BCS-Hubbard Hamiltonian, which is rigorously studied at equilibrium in [3] in order to understand the possible thermodynamic impact of the Coulomb repulsion on ( $s$-wave) superconductivity. An interesting mathematical outcome of [3] on the strong-coupling BCS-Hubbard Hamiltonian is the existence of a superconductorMott insulator phase transition, like in cuprates which must be doped to become superconductors.

The results of [3] are based on an exact study of the phase diagram of the strong-coupling BCS-Hubbard model defined, in a cubic box $\Lambda_{L} \doteq\{\mathbb{Z} \cap[-L, L]\}^{d}(d \in \mathbb{N})$ of volume $\left|\Lambda_{L}\right|$ for $L \in \mathbb{N}_{0}$, by the Hamiltonian

$$
\mathrm{H}_{L} \doteq \sum_{x \in \Lambda_{L}}\left(2 \lambda n_{x, \uparrow} n_{x, \downarrow}-\mu\left(n_{x, \uparrow}+n_{x, \downarrow}\right)-h\left(n_{x, \uparrow}-n_{x, \downarrow}\right)\right)-\frac{\gamma}{\left|\Lambda_{L}\right|} \sum_{x, y \in \Lambda_{L}} a_{x, \uparrow}^{*} a_{x, \downarrow}^{*} a_{y, \downarrow} a_{y, \uparrow}
$$

for real parameters $\mu, h \in \mathbb{R}$ and $\lambda, \gamma \geq 0$. The operator $a_{x, \mathrm{~s}}^{*}$ (resp. $a_{x, \mathrm{~s}}$ ) creates (resp. annihilates) a fermion with spin $\mathrm{s} \in\{\uparrow, \downarrow\}$ at lattice position $x \in \mathbb{Z}^{d}, d=1,2,3, \ldots$, whereas $n_{x, \mathrm{~s}} \doteq a_{x, \mathrm{~s}}^{*} a_{x, \mathrm{~s}}$ is the particle number operator at position $x$ and spin s. They are linear operators acting on the fermion Fock space $\mathcal{F}_{\Lambda_{L}}$, where

$$
\mathcal{F}_{\Lambda} \doteq \bigwedge \mathbb{C}^{\Lambda \times\{\uparrow, \downarrow\}} \equiv \mathbb{C}^{2^{\Lambda \times\{\uparrow, \downarrow\}}}
$$

for any $\Lambda \subseteq \mathbb{Z}^{d}$ and $d \in \mathbb{N}$. The first term of the right-hand side of (3) represents the (screened) Coulomb repulsion as in the celebrated Hubbard model. The second term corresponds to the strong-coupling limit of the kinetic energy, also called "atomic limit" in the context of the Hubbard model. The third term is the interaction between spins and the external magnetic field $h$. The last term is the BCS interaction written in the $x$-space since

$$
\frac{\gamma}{\left|\Lambda_{L}\right|} \sum_{x, y \in \Lambda_{L}} a_{x, \uparrow}^{*} a_{x, \downarrow}^{*} a_{y, \downarrow} a_{y, \uparrow}=\frac{\gamma}{\left|\Lambda_{L}\right|} \sum_{k, q \in \Lambda_{L}^{*}} \tilde{a}_{k, \uparrow}^{*} \tilde{a}_{-k, \downarrow}^{*} \tilde{a}_{q, \downarrow} \tilde{a}_{-q, \uparrow} .
$$

See (2) with $\gamma_{k, q}=\gamma>0$. This homogeneous BCS interaction should be seen as a long-range effective interaction, the precise mediators of which are not relevant, i.e., they could be phonons, as in conventional type I superconductors, or anything else.

\footnotetext{
${ }^{1}$ The positivity of $\gamma_{k, q}$ imposes constraints on the choice of the function $f$.
} 


\section{Approximating Hamiltonians}

The thermodynamic impact of the Coulomb repulsion on s-wave superconductors is analyzed in [3], via a rigorous study of equilibrium and ground states of the strong-coupling BCS-Hubbard Hamiltonian: An Hamiltonian like $\mathrm{H}_{L}$ defines in the thermodynamic limit $L \rightarrow \infty$ a free-energy density functional on a suitable set of states of the CAR algebra of the lattice $\mathbb{Z}^{d}$. See [3, Section 6.2] for more details. Minimizers $\omega$ of the free-energy density are called equilibrium states of the model and, for any $L \in \mathbb{N}_{0}$, the Gibbs states $\omega^{(L)}$, defined on the algebra $\mathcal{B}\left(\mathcal{F}_{\Lambda_{L}}\right)$ of linear operators acting on the fermion Fock space $\mathcal{F}_{\Lambda_{L}}(4)$ by

$$
\omega^{(L)}(A) \doteq \operatorname{Trace}_{\mathcal{F}_{\Lambda_{L}}}\left(A \frac{\mathrm{e}^{-\beta \mathrm{H}_{L}}}{\operatorname{Trace}_{\mathcal{F}_{\Lambda_{L}}}\left(\mathrm{e}^{\left.-\beta \mathrm{H}_{L}\right)}\right.}\right), \quad A \in \mathcal{B}\left(\mathcal{F}_{\Lambda_{L}}\right),
$$

at inverse temperature $\beta>0$, converges ${ }^{2}$ in the thermodynamic limit $L \rightarrow \infty$ to a well-defined equilibrium state. The important point in such an analysis is the study of a variational problem over complex numbers: By the so-called approximating Hamiltonian method [8-10] one uses an approximation of the Hamiltonian, which, in the case of the strong-coupling BCS-Hubbard Hamiltonian, is equal to the $c$-dependent Hamiltonian

$$
\mathrm{H}_{L}(c) \doteq \sum_{x \in \Lambda_{L}}\left(2 \lambda n_{x, \uparrow} n_{x, \downarrow}-\mu\left(n_{x, \uparrow}+n_{x, \downarrow}\right)-h\left(n_{x, \uparrow}-n_{x, \downarrow}\right)-\gamma\left(c a_{x, \uparrow}^{*} a_{x, \downarrow}^{*}+\bar{c} a_{x, \downarrow} a_{x, \uparrow}\right)\right)
$$

with $c \in \mathbb{C}$. The main advantage of using this $c$-dependent Hamiltonian, in comparison with $\mathrm{H}_{L}$, is the fact that it is a sum of shifts of the same on-site operator. For an appropriate choice of (order) parameter $c \in \mathbb{C}$, it leads to the exact thermodynamics of the strong-coupling BCSHubbard model, in the limit $L \rightarrow \infty$ : At inverse temperature $\beta>0$,

$$
\lim _{L \rightarrow \infty} \frac{1}{\beta\left|\Lambda_{L}\right|} \ln \operatorname{Trace}_{\mathcal{F}_{\Lambda_{L}}}\left(\mathrm{e}^{-\beta \mathrm{H}_{L}}\right)=\sup _{c \in \mathbb{C}}\left\{-\gamma|c|^{2}+\lim _{L \rightarrow \infty}\left\{\frac{1}{\beta\left|\Lambda_{L}\right|} \ln \operatorname{Trace}_{\mathcal{F}_{\Lambda_{L}}}\left(\mathrm{e}^{-\beta \mathrm{H}_{L}(c)}\right)\right\}\right\}
$$

and the (exact) Gibbs state $\omega^{(L)}$ converges $^{3}$ to a convex combination ${ }^{4}$ of the thermodynamic limit $L \rightarrow \infty$ of the (approximating) Gibbs state $\omega^{(L, \mathfrak{d})}$ defined by

$$
\omega^{(L, \mathfrak{d})}(A) \doteq \operatorname{Trace}_{\mathcal{F}_{\Lambda_{L}}}\left(A \frac{\mathrm{e}^{-\beta \mathrm{H}_{L}(\mathfrak{d})}}{\operatorname{Trace}_{\mathcal{F}_{\Lambda_{L}}}\left(\mathrm{e}^{-\beta \mathrm{H}_{L}(\mathfrak{d})}\right)}\right), \quad A \in \mathcal{B}\left(\mathcal{F}_{\Lambda_{L}}\right)
$$

the complex number $\mathfrak{d} \in \mathbb{C}$ being a solution to the variational problem (8). Since $\gamma \geq 0$, this can be heuristically be seen from the inequality

$$
\gamma\left|\Lambda_{L}\right||c|^{2}+\mathrm{H}_{L}(c)-\mathrm{H}_{L}=\gamma\left(\mathfrak{c}_{0}^{*}-\sqrt{\left|\Lambda_{L}\right|} \bar{c}\right)\left(\mathfrak{c}_{0}-\sqrt{\left|\Lambda_{L}\right|} c\right) \geq 0,
$$

where

$$
\mathfrak{c}_{0} \doteq \frac{1}{\sqrt{\left|\Lambda_{L}\right|}} \sum_{x \in \Lambda_{L}} a_{x, \downarrow} a_{x, \uparrow}
$$

\footnotetext{
${ }^{2}$ In the weak* topology.

${ }^{3}$ In the weak* topology.

${ }^{4}$ More precisely, it converges to the barycenter of a Choquet measure.
} 
(resp. $\mathfrak{c}_{0}^{*}$ ) annihilates (resp. creates) one Cooper pair within the condensate, i.e., in the zero-mode for fermion pairs. This suggests the proven fact [3, Theorem 3.1] that

$$
|\mathfrak{d}|^{2}=\lim _{L \rightarrow \infty} \frac{\omega^{(L)}\left(\mathfrak{c}_{0}^{*} \mathfrak{c}_{0}\right)}{\left|\Lambda_{L}\right|}
$$

for any ${ }^{5} \mathfrak{d} \in \mathbb{C}$ solution to the variational problem (8). The parameter $\left[* *|\mathfrak{d}|^{2 * *}\right]$ is the condensate density of Cooper pairs and so, $[* * \mathfrak{d} \neq 0 * *]$ corresponds to the existence of a superconducting phase, which is shown to exist for sufficiently large $\gamma \geq 0$. See also [3, Figs. 1,2,3].

\section{Dynamical Problem in the Thermodynamic Limit}

As is usual, a Hamiltonian like the strong-coupling BCS-Hubbard model drives a dynamics in the Heisenberg picture of quantum mechanics: The corresponding time-evolution is, for $L \in \mathbb{N}_{0}$, a continuous group $\left\{\tau_{t}^{(L)}\right\}_{t \in \mathbb{R}}$ of $*$-automorphisms of the algebra $\mathcal{B}\left(\mathcal{F}_{\Lambda_{L}}\right)$ of linear operators acting on the Fermion Fock space $\mathcal{F}_{\Lambda_{L}}$ (see (4)), defined by

$$
\tau_{t}^{(L)}(A) \doteq \mathrm{e}^{i t \mathrm{H}_{L}} A \mathrm{e}^{-i t \mathrm{H}_{L}}, \quad A \in \mathcal{B}\left(\mathcal{F}_{\Lambda_{L}}\right), t \in \mathbb{R} .
$$

The generator of this time evolution is the linear operator $\delta_{L}$ defined on $\mathcal{B}\left(\mathcal{F}_{\Lambda_{L}}\right)$ by

$$
\delta_{L}(A) \doteq i\left[\mathrm{H}_{L}, A\right] \doteq i\left(\mathrm{H}_{L} A-A \mathrm{H}_{L}\right), \quad A \in \mathcal{B}\left(\mathcal{F}_{\Lambda_{L}}\right)
$$

If $\gamma=0$ then it is well-known that the thermodynamic limit of $\left\{\tau_{t}^{(L)}\right\}_{t \in \mathbb{R}}$ exists as a strongly continuous group $\left\{\tau_{t}\right\}_{t \in \mathbb{R}}$ of $*$-automorphisms of the CAR algebra of the infinite lattice. If $\gamma>0$ then the situation is not that obvious. A first guess is to approximate $\left\{\tau_{t}^{(L)}\right\}_{t \in \mathbb{R}}$ by $\left\{\tau_{t}^{(L, c)}\right\}_{t \in \mathbb{R}}$, where

$$
\tau_{t}^{(L, c)}(A) \doteq \mathrm{e}^{i t \mathrm{H}_{L}(c)} A \mathrm{e}^{-i t \mathrm{H}_{L}(c)}, \quad A \in \mathcal{B}\left(\mathcal{F}_{\Lambda_{L}}\right), t \in \mathbb{R},
$$

for any $L \in \mathbb{N}_{0}$ and some complex number $c \in \mathbb{C}$. In this case, the linear operator

$$
\delta_{L, c}(A) \doteq i\left[\mathrm{H}_{L}(c), A\right], \quad A \in \mathcal{B}\left(\mathcal{F}_{\Lambda_{L}}\right),
$$

is the generator of the dynamics $\left\{\tau_{t}^{(L, c)}\right\}_{t \in \mathbb{R}}$. A natural choice for $c \in \mathbb{C}$ would be a solution to the variational problem (8), but what about if the solution is not unique? As a matter of fact, as explained in [1, Section 4.3], in the thermodynamic limit $L \rightarrow \infty$, the finite-volume dynamics $\left\{\tau_{t}^{(L)}\right\}_{t \in \mathbb{R}}$ does not converge within the CAR $C^{*}$-algebra of the infinite lattice for $\gamma>0$, even if $\mathfrak{d}=0$ would be the unique solution to the variational problem (8)! Observe, moreover, that the variational problem (8) depends on the temperature whereas the time evolution (12) does not.

The validity of the Bogoliubov approximation (13) with respect to the full dynamics (12) was an open question that Thirring and Wehrl [11,12] solve in 1967 for the special case $\left.\mathrm{H}_{L}\right|_{\mu=\lambda=h=0}$, which is an exactly solvable permutation-invariant model for any $\gamma \in \mathbb{R}$. An attempt to generalize Thirring and Wehrl's results to a general class of fermionic models, including the BCS theory, has been done in 1978 [13], but at the cost of technical assumptions that are difficult to verify in practice. This research direction has been strongly developed by many authors until 1992, see [14-33]. All these papers study dynamical properties of permutation-invariant quantum-spin systems with mean-field interactions. Our results $[1,2]$ represent a significant generalization of such previous results to possibly non-permutation-invariant lattice-fermion or quantum-spin systems. To understand what's going on in the infinite-volume dynamics, we now come back to our pedagogical example, that is, the strong-coupling BCS-Hubbard model.

\footnotetext{
${ }^{5}$ This implies that any solution $|d|$ to the variational problem (8) must have the same absolute value.
} 


\section{Self-Consistency Equations}

Instead of considering the Heisenberg picture, let us consider now the Schrödinger picture of quantum mechanics. In this case, recall that, at fixed $L \in \mathbb{N}_{0}$, a finite-volume state $\rho^{(L)}$ is defined by

$$
\rho^{(L)}(A) \doteq \operatorname{Trace}_{\mathcal{F}_{\Lambda_{L}}}\left(\mathrm{~d}^{(L)} A\right), \quad A \in \mathcal{B}\left(\mathcal{F}_{\Lambda_{L}}\right),
$$

for a uniquely defined positive operator $\mathrm{d}^{(L)} \in \mathcal{B}\left(\mathcal{F}_{\Lambda_{L}}\right)$ satisfying Trace $\mathcal{F}_{\Lambda_{L}}\left(\mathrm{~d}^{(L)}\right)=1$ and named the density matrix of $\rho^{(L)}$. Compare with (6) and (9). At $L \in \mathbb{N}_{0}$, the time evolution of any finite-volume state is

$$
\rho_{t}^{(L)} \doteq \rho^{(L)} \circ \tau_{t}^{(L)}, \quad t \in \mathbb{R}
$$

which corresponds to a time-dependent density matrix equal to $\mathrm{d}_{t}^{(L)}=\tau_{-t}^{(L)}\left(\mathrm{d}^{(L)}\right)$.

The thermodynamic limit of (15) for periodic initial states can be explicitly computed, as explained in [4, Section 4.3.2]. It refers to a non-linear state-dependent dynamics related to selfconsistency: By (4), $\mathcal{B}\left(\mathcal{F}_{\{0\}}\right)$ can be identified with the set Mat $(4, \mathbb{C})$ of complex $4 \times 4$ matrices, in some orthonormal basis ${ }^{6}$. For any continuous family $\omega \doteq\left(\omega_{t}\right)_{t \in \mathbb{R}}$ of states acting on $\mathcal{B}\left(\mathcal{F}_{\{0\}}\right)$, we define the finite-volume non-autonomous dynamics $\left(\tau_{t, s}^{(L, \omega)}\right)_{s, t \in \mathbb{R}}$ by the Dyson-Phillips series

$$
\tau_{t, s}^{(L, \omega)} \equiv \cdots \exp \left(\int_{s}^{t} \delta_{L}^{\omega_{u}} \mathrm{~d} u\right) " \doteq \mathbf{1}_{\mathcal{B}\left(\mathcal{F}_{\Lambda_{L}}\right)}+\sum_{k \in \mathbb{N}} \int_{s}^{t} \mathrm{~d} t_{1} \cdots \int_{s}^{t_{k-1}} \mathrm{~d} t_{k} \delta_{L}^{\omega_{t_{k}}} \circ \cdots \circ \delta_{L}^{\omega_{t_{1}}}
$$

acting on $\mathcal{B}\left(\mathcal{F}_{\Lambda_{L}}\right)$ for any $s, t \in \mathbb{R}$, with $\mathbf{1}_{\mathcal{B}\left(\mathcal{F}_{\Lambda_{L}}\right)}$ being the identity mapping of $\mathcal{B}\left(\mathcal{F}_{\Lambda_{L}}\right)$ and where $\delta_{L}^{\rho}$ is the generator of the group $\left\{\tau_{t}^{(L, c)}\right\}_{t \in \mathbb{R}}$, defined by (14) for $c=\rho\left(a_{0, \uparrow} a_{0, \downarrow}\right)$. Compare with (11) and (10). Note that, for every continuous family $\omega \doteq\left(\omega_{t}\right)_{t \in \mathbb{R}}$ of on-site (even) states acting on $\mathcal{B}\left(\mathcal{F}_{\{0\}}\right), s, t \in \mathbb{R}, L_{0} \in \mathbb{N}_{0}$ and all integers $L \geq L_{0}$,

$$
\tau_{t, s}^{(L, \omega)}(A)=\tau_{t, s}^{\left(L_{0}, \omega\right)}(A), \quad A \in \mathcal{B}\left(\mathcal{F}_{\Lambda_{L_{0}}}\right) .
$$

It follows that the family $\left\{\tau_{t, s}^{(L, \omega)}\right\}_{s, t \in \mathbb{R}}$ strongly converges in the thermodynamic limit $L \rightarrow \infty$ to a strongly continuous two-parameter family $\left\{\tau_{t, s}^{\omega}\right\}_{s, t \in \mathbb{R}}$ of $*$-automorphisms of the CAR algebra of the lattice. With these observations, we are in a position to give the self-consistency equations: By [4, Eq. (19)], for any fixed initial (even) state $\rho$ on $\mathcal{B}\left(\mathcal{F}_{\{0\}}\right)$ at $t=0$, there is a unique family $(\varpi(t, \rho))_{t \in \mathbb{R}}$ of (on-site) states acting on $\mathcal{B}\left(\mathcal{F}_{\{0\}}\right)$ such that

$$
\varpi(t, \rho)=\rho \circ \tau_{t, 0}^{\varpi(\cdot, \rho)}, \quad t \in \mathbb{R} .
$$

Observe that (17) is an equation on a finite-dimensional space, see (4).

\section{Infinite-Volume Dynamics of Product States}

For simplicity, as initial state (at $t=0$ ), take a finite-volume product ${ }^{7}$ state $\rho^{(L)} \doteq \otimes_{\Lambda_{L}} \rho$ associated with an even ${ }^{8}$ state $\rho$ on $\mathcal{B}\left(\mathcal{F}_{\{0\}}\right)$. An example of finite-volume product states is given by the

\footnotetext{
${ }^{6}$ For instance, $(1,0,0,0)$ is the vacuum; $(0,1,0,0)$ and $(0,0,1,0)$ correspond to one fermion with spin $\uparrow$ and $\downarrow$, respectively; $(0,0,0,1)$ refers to two fermions with opposite spins.

${ }^{7}$ The product state $\rho^{(L)}$ is (well-) defined by $\rho^{(L)}\left(\alpha_{x_{1}}\left(A_{1}\right) \cdots \alpha_{x_{n}}\left(A_{n}\right)\right)=\rho\left(A_{1}\right) \cdots \rho\left(A_{n}\right)$ for all $A_{1}, \ldots, A_{n} \in$ $\mathcal{B}\left(\mathcal{F}_{\{0\}}\right)$ and all $x_{1}, \ldots, x_{n} \in \Lambda_{L}$ such that $x_{i} \neq x_{j}$ for $i \neq j$, where $\alpha_{x_{j}}\left(A_{j}\right) \in \mathcal{B}\left(\mathcal{F}_{\left\{x_{j}\right\}}\right)$ is the $x_{j}$-translated copy of $A_{j}$ for all $j \in\{1, \ldots, n\}$.

${ }^{8}$ Even states are the physically relevant ones. Even means that the expectation value of any odd monomials in $\left\{a_{0, \mathrm{~s}}^{*}, a_{0, \mathrm{~s}}\right\}_{\mathrm{s} \in\{\uparrow, \downarrow\}}$ with respect to the on-site state $\rho$ is zero.
} 
approximating Gibbs states (9). Then, in this case, as explained in [4, Section 4.4], for any $t \in \mathbb{R}$, $L_{0} \in \mathbb{N}_{0}$ and $A \in \mathcal{B}\left(\mathcal{F}_{\Lambda_{L_{0}}}\right)$, one has that

$$
\rho_{t}(A) \doteq \lim _{L \rightarrow \infty} \rho_{t}^{(L)}(A)=\lim _{L \rightarrow \infty} \rho^{(\infty)} \circ \tau_{t}^{(L)}(A)=\rho^{(\infty)} \circ \tau_{t, 0}^{\varpi(\cdot, \rho)}(A)
$$

with $\rho_{t}^{(L)}, \varpi(\cdot, \rho)$ being respectively defined by (15) and (17) and where $\rho^{(\infty)} \doteq \otimes_{\mathbb{Z}^{d}} \rho$ is the (infinite-volume) product state associated with the even state $\rho$ on $\mathcal{B}\left(\mathcal{F}_{\{0\}}\right)$.

For any $t \in \mathbb{R}$, the limit state $\rho_{t}$ is again a product state and hence, it is completely determined by its restriction to the single lattice site $(0, \ldots, 0) \in \mathbb{Z}^{d}$, that is, by the on-site state $\varpi(t, \rho)$ for all $t \in \mathbb{R}$. Below, we give the explicit expressions for the time evolution of the most important physical quantities related to this model, in this situation for any time $t \in \mathbb{R}$ :

(i) Electron density:

$$
\mathrm{d}(\rho) \doteq \rho\left(n_{0, \uparrow}+n_{0, \downarrow}\right)=\rho_{t=0}\left(n_{0, \uparrow}+n_{0, \downarrow}\right)=\rho_{t}\left(n_{0, \uparrow}+n_{0, \downarrow}\right) \in[0,2] .
$$

(ii) Magnetization density:

$$
\mathrm{m}(\rho) \doteq \rho\left(n_{0, \uparrow}-n_{0, \downarrow}\right)=\rho_{t=0}\left(n_{0, \uparrow}-n_{0, \downarrow}\right)=\rho_{t}\left(n_{0, \uparrow}-n_{0, \downarrow}\right) \in[-1,1] .
$$

(iii) Coulomb correlation density:

$$
\mathrm{w}(\rho) \doteq \rho\left(n_{0, \uparrow} n_{0, \downarrow}\right)=\rho_{t=0}\left(n_{0, \uparrow} n_{0, \downarrow}\right)=\rho_{t}\left(n_{0, \uparrow} n_{0, \downarrow}\right) \in[0,1] .
$$

(iv) Cooper field and condensate densities:

$$
\begin{aligned}
& \rho_{t}\left(a_{0, \downarrow} a_{0, \uparrow}\right)=\sqrt{\kappa(\rho)} \mathrm{e}^{i(t \nu(\rho)+\theta(\rho))} \quad \text { with } \quad \nu(\rho) \doteq 2(\mu-\lambda)+\gamma(1-\mathrm{d}(\rho)) \\
& \text { and } \kappa(\rho) \in[0,1], \theta(\rho) \in[-\pi, \pi) \text { such that } \rho\left(a_{0, \downarrow} a_{0, \uparrow}\right)=\sqrt{\kappa(\rho)} \mathrm{e}^{i \theta(\rho)} .
\end{aligned}
$$

See [4, Lemma 1]. In the special case $\lambda=0$, i.e., without the Hubbard interaction, (i)-(iv) reproduce the results of [21, Section A] on the strong-coupling BCS model, written in that paper as a permutation-invariant quantum-spin model.

From Assertions (i)-(iv) observe that we recover the equation of a symmetric rotor in classical mechanics: Fix an even on-site state $\rho$. For any $t \in \mathbb{R}$, define the $3 \mathrm{D}$ vector $\left(\Omega_{1}(t), \Omega_{2}(t), \Omega_{3}(t)\right)$ by

$$
\rho_{t}\left(a_{0, \downarrow} a_{0, \uparrow}\right)=\Omega_{1}(t)+i \Omega_{2}(t) \quad \text { and } \quad \Omega_{3}(t) \doteq 2(\mu-\lambda)+\gamma\left(1-\rho_{t}\left(n_{0, \uparrow}+n_{0, \downarrow}\right)\right) \text {. }
$$

Then, this $3 \mathrm{D}$ vector satisfies, for any time $t \in \mathbb{R}$, the following system of ODEs:

$$
\left\{\begin{array}{l}
\dot{\Omega}_{1}(t)=-\Omega_{3}(t) \Omega_{2}(t), \\
\dot{\Omega}_{2}(t)=\Omega_{3}(t) \Omega_{1}(t), \\
\dot{\Omega}_{3}(t)=0
\end{array}\right.
$$

which describes the time evolution of the angular momentum of a symmetric rotor in classical mechanics. 
In fact, by seeing quantum states as elements of a state space in classical mechanics, this dynamics can be written in terms of Poisson brackets, i.e., as some Liouville's equation of classical mechanics, as proven in [1, Corollary 6.11] for any translation invariant long-range models. Moreover, [1,2] show that long-range dynamics in infinite volume are equivalent to intricate combinations of classical and quantum short-range dynamics, opening new theoretical perspectives, as explained in [34]. This phenomenon is a direct consequence of the highly non-local character of long-range, or mean-field, interactions.

Assertions (i)-(iv) lead to the exact dynamics of a physical system prepared in a product state at initial time, driven by the strong-coupling BCS-Hubbard Hamiltonian. This set of states is still [**restrictive**] and our results [1,2] go beyond this simple case, by allowing us to consider general periodic states as initial states, in contrast with all previous results on lattice Fermi, or quantum-spin, systems with long-range, or mean-field, interactions. See [4, Section 2.6].

Acknowledgments: This work is supported by CNPq (308337/2017-4), FAPESP (2017/223409), as well as by the Basque Government through the grant IT641-13 and the BERC 2018-2021 program, and by the Spanish Ministry of Science, Innovation and Universities: BCAM Severo Ochoa accreditation SEV-2017-0718, MTM2017-82160-C2-2-P.

\section{References}

[1] Bru J.-B., de Siqueira Pedra W. , Classical Dynamics Generated by Long-Range Interactions for Lattice Fermions and Quantum Spins, J. Math. Anal. Appl. (2020) https://doi.org/10.1016/j.jmaa.2020.124434.

[2] Bru J.-B., de Siqueira Pedra W. Quantum Dynamics Generated by Long-Range Interactions for Lattice Fermions and Quantum Spins, J. Math. Anal. Appl. (2020) https://doi.org/10.1016/j.jmaa.2020.124517.

[3] Bru J.-B., de Siqueira Pedra W. Rev. Math. Phys. 2010. V. 22(3) P. 233-303.

[4] Bru J.-B., de Siqueira Pedra W. Macroscopic Long-Range Dynamics of Fermions and Quantum Spins on the Lattice - An Introduction. Preprint 2019. See mp_arc 19-399.

[5] Metzner W., Castellani C., Di Castro C. Advances in Physics 1998 V. 47(3) P. 317-445.

[6] Thouless D. J. The Quantum Mechanics of Many-Body Systems. Second Edition. Academic Press, New York, 1972.

[7] Bogoliubov N. N., Tolmachev V.V., Shirkov D.V. A New Method in the Theory of Superconductivity. Academy of Sciences Press, Moscow, 1958 or Consult.Bureau, Inc., N.Y., Chapman Hall Ltd., London, 1959.

[8] Bogoliubov Jr. N.N., Brankov J.G., Zagrebnov V.A., Kurbatov A.M. and Tonchev N.S. Metod approksimiruyushchego gamil'toniana v statisticheskoi fizike'. Sofia: Izdat. Bulgar. Akad. Nauk $^{10}, 1981$.

\footnotetext{
${ }^{9}$ The Approximating Hamiltonian Method in Statistical Physics.

${ }^{10}$ Publ. House Bulg. Acad. Sci.
} 
[9] Bogoliubov Jr. N.N., Brankov J.G., Zagrebnov V.A., Kurbatov A.M., Tonchev N.S. Russ. Math. Surv. 1984. V. 39 P. 1-50.

[10] Brankov J.G., Danchev D.M., Tonchev N.S. Theory of Critical Phenomena in Finite-size Systems: Scaling and Quantum Effects. Singapore-New Jersey-London-Hong Kong, Word Scientific, 2000.

[11] Thirring W., Wehrl A. Comm. Math. Phys. 1967. V. 4 P. 303-314.

[12] Thirring W. Comm. Math. Phys. 1968. V. 7 P. 181-189.

[13] Hemmen L. van Bortachritte der Physik 1978. V. 26 P. 397-439; Section III.

[14] Bóna P. acta phys sIov. 1975. V. 25(1) P. 3-21.

[15] Sewell G. L. Lett. Math. Phys. 1982. V. 6 P. 209-213.

[16] Rieckers A. J. Math. Phys. 1984. V. 25 P. 2593-2601.

[17] Morchio G., Strocchi F. J. Math. Phys. 1987. V. 28 P. 622-635.

[18] Bóna P. Czech. J. Phys. B 1987. V. 37 P. 482-491.

[19] Duffner E., Rieckers A. Z. Naturforsch. 1988. V. 43a P. 521-532.

[20] Bóna P. J. Math. Phys. 1988. V. 29 P. 2223-2235.

[21] Bóna P. J. Math. Phys. 1989. V. 30 P. 2994-3007.

[22] Bóna P. On Symmetries in Mean-Field Theories, in Selected Topics in Quantum Field Theory and Mathematical Physics, Eds. Niederle J., Fischer J., World Scientic, Singapore, 1990.

[23] Unnerstall T. J. Math. Phys. 1990. V. 31 P. 680-688.

[24] Unnerstall T. Comm. in Math. Phys. 1990. V. 130 P. 237-255.

[25] Unnerstall T. Lett. Math. Phys. 1990. V. 20 P. 183-187.

[26] Bóna P. Quantum Mechanics with Mean-Field Backgrounds, Preprint No. Ph10-91, Comenius University, Faculty of Mathematics and Physics, Bratislava, October 1991.

[27] Duffield N.G. Helvet. Phys. Acta. 1991. V. 64 P. 610-632.

[28] Bagarello F., Morchio G. J. Stat. Phys. 1992. V. 66 P. 849-866.

[29] Duffield N.G., Werner R.F. Helvetica Physica Acta 1992. V. 65 P. 1016-1054.

[30] Duffield N.G., Werner R.F. Rev. Math. Phys. 1992. V. 4 P. 383-424.

[31] Duffield N.G., Roos H., Werner R.F. Ann. Inst. H. Poincaré Phys. Théor. 1992. V. 56 P. 143-186.

[32] Duffield N.G., Werner R.F. Rev. Math. Phys. 1992. V. 4(3) P. 383-424. 
[33] Duffield N.G., Werner R.F. in: Stochastics and quantum mechanics (Swansea, 1990), Eds. Truman A. and Davies I.M. World Sci. Publishing, River Edge, NJ 1992. P. 115-129

[34] Bru J.-B. and de Siqueira Pedra W., Classical Dynamics from Self-Consistency Equations in Quantum Mechanics - Extended Version. Preprint arXiv:2009.04969 (2020). 